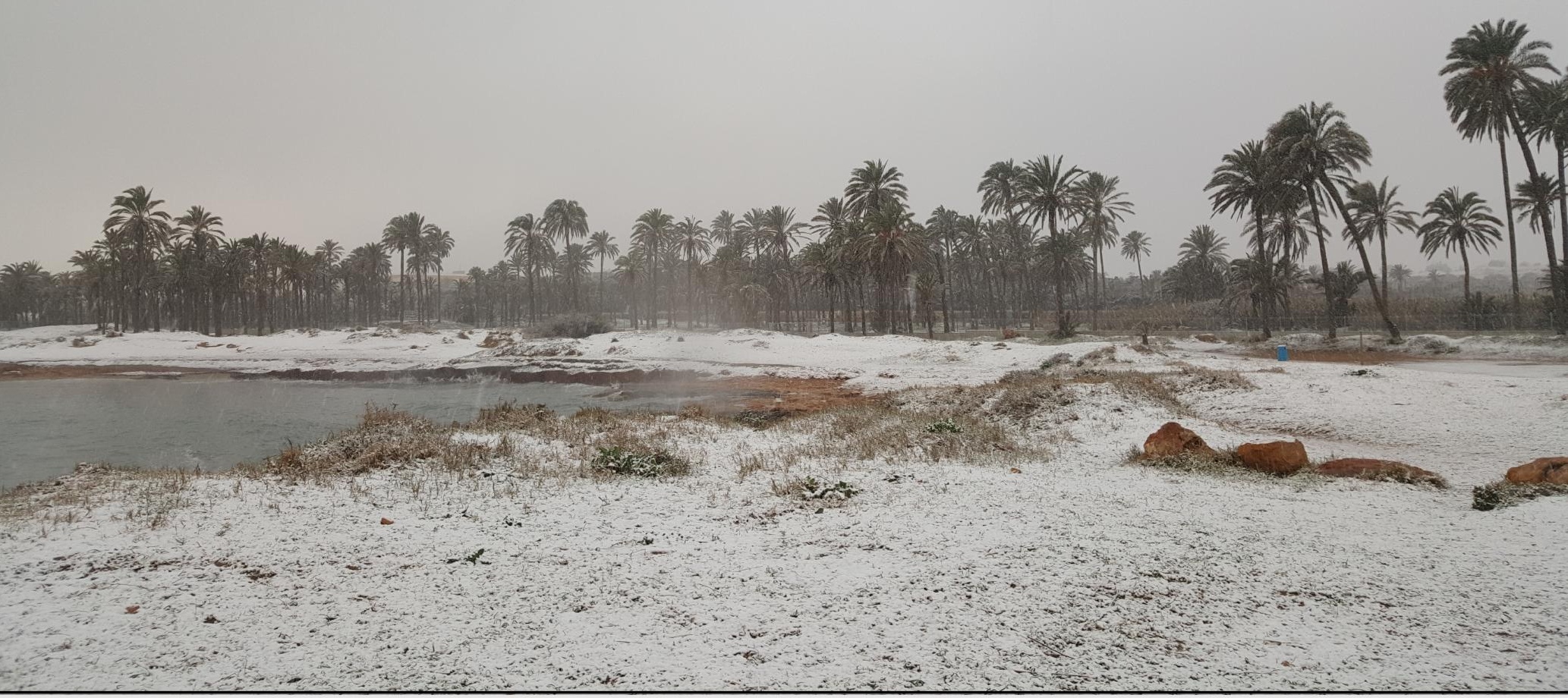

\title{
Temporal mediterráneo de lluvia, nieve y viento (Ene 2017)
}

\author{
Agustí JANSÀ ClaR \\ Grupo de Meteorología, Departamento de Física, Universitat de les Illes Balears
}

En Valencia, la nieve cubrió playas en la Costa Blanca como las alicantinas de Denia, Orihuela y
Campoamor, donde cuajó, y dejó incomunicada la localidad costera de Jávea. Ese paisaje no se
veía en el municipio desde 1983. También en Torrevieja se dejaron ver los copos 103 años después.

La nieve llega a playas del Mediterráneo, 19-01-2017 - MERCEDES LODEIRO, La Voz de Galicia

Lo que se había iniciado como una fuerte irrupción fría entre los días 16 y 17 de enero de 2017, derivó hacia la formación de una borrasca mediterránea, bastante intensa y de compleja evolución, que dio lugar a un temporal importante de lluvia, nieve, viento y oleaje alto, afectando principalmente a la Comunidad Valenciana y a las Illes Balears. Algunas cantidades de precipitación fueron muy notables, sobre todo teniendo en cuenta la época, que en el Mediterráneo no es la de mayores lluvias. El viento fue también fuerte y el oleaje batió récords de altura en la zona afectada, incluso generando daños estructurales. Las predicciones basadas en sistemas de predicción por conjuntos apuntaron en la dirección correcta, orientando adecuadamente para la toma de decisiones, con claras diferencias según el plazo y con ciertas insuficiencias, incluso a plazos relativamente cortos. Este episodio se estudia también, desde otro punto de vista, en Bermejo [1].

Palabras clave: sistemas de predicción por conjuntos aplicados a la predicción de corto y medio plazo, predicción probabilista, temporal mediterráneo de lluvia-nieve-viento enero 2017. 


\subsection{Un episodio con lluvia muy} copiosa, nieve, viento fuerte, mar alta y ciclogénesis importante

Un año después de una insólita sequía invernal en el Mediterráneo español (noviembre y diciembre de 2015 y enero de 2016), en diciembre de 2016 y enero de 2017 se produjeron varios temporales mediterráneos de gran intensidad, con lluvias y vientos, si no insólitos, sí al menos inusuales, por fuertes, sobre todo teniendo en cuenta la época.

Uno de esos temporales tuvo lugar en dos fases, entre los días 19 y 24 de enero de 2017. El día 19 hubo, sobre todo, lluvia fuerte, particularmente en la Comunidad Valenciana, aunque también las Illes Balears se vieron afectadas. El día 20 hubo una cierta transición. El día 21, con amortiguamiento progresivo después, la lluvia intensa y el fuerte viento afectaron a Illes Balears y a toda el área marítima comprendida entre el Archipiélago y la península ibérica. El día 22 las lluvias más importantes se habían desplazado al norte de Catalunya.

Como ocurre con muchos temporales mediterráneos importantes, éste estuvo ligado a la formación, o intensificación autóctona, de una depresión en el Mediterráneo. En realidad, a la vista de las Figuras 52.1 y 52.2, podríamos hablar de una depresión pulsante, centrada entre la Península y la costa africana. El día 19 hay un primer intento de formación de depresión, pero apenas hay desarrollo y la duración es de horas, solamente, desapareciendo pronto la modesta depresión formada. Desde las 06 UTC del día 20 a las 06 UTC del 21, en cambio, la ciclogénesis es importante, con una caída de la presión central de $10 \mathrm{hPa}$ en 24 horas. La depresión se mantiene potente durante el día 21 y va decayendo a lo largo del 22, aunque todavía será reconocible durante un tiempo.

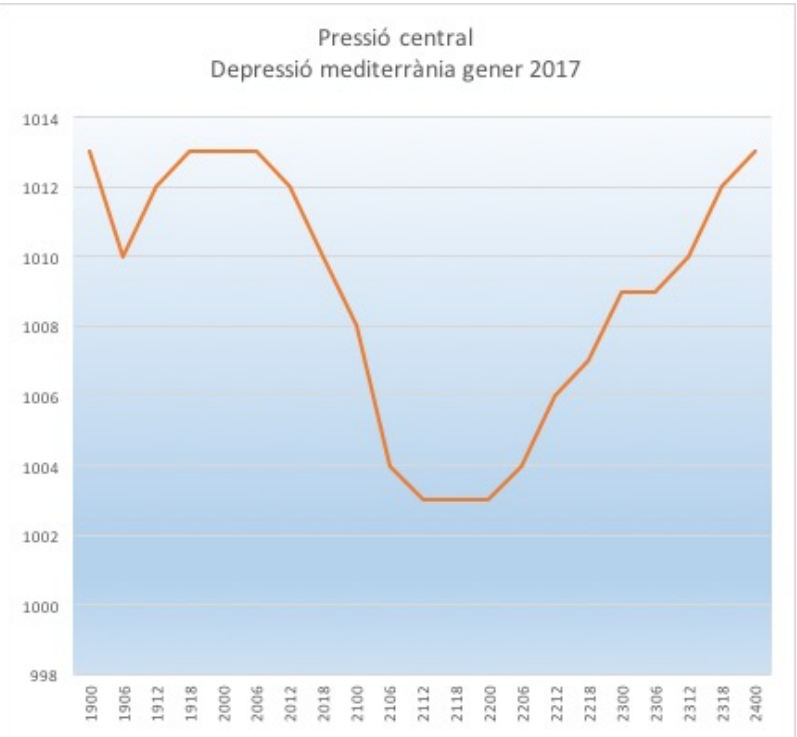

Figura 52.1: Evolución temporal de la presión central en la depresión mediterránea de enero de 2017, relacionada con el temporal de lluvia y viento del 19 al 24 del citado mes. La gráfica ha sido obtenida a partir de análisis y/o campos previstos a corto plazo de presión al nivel del mar, del modelo Global Forecasting System (GFS) de la National Oceanic and Atmospheric Administration (NOAA) [3, 4].
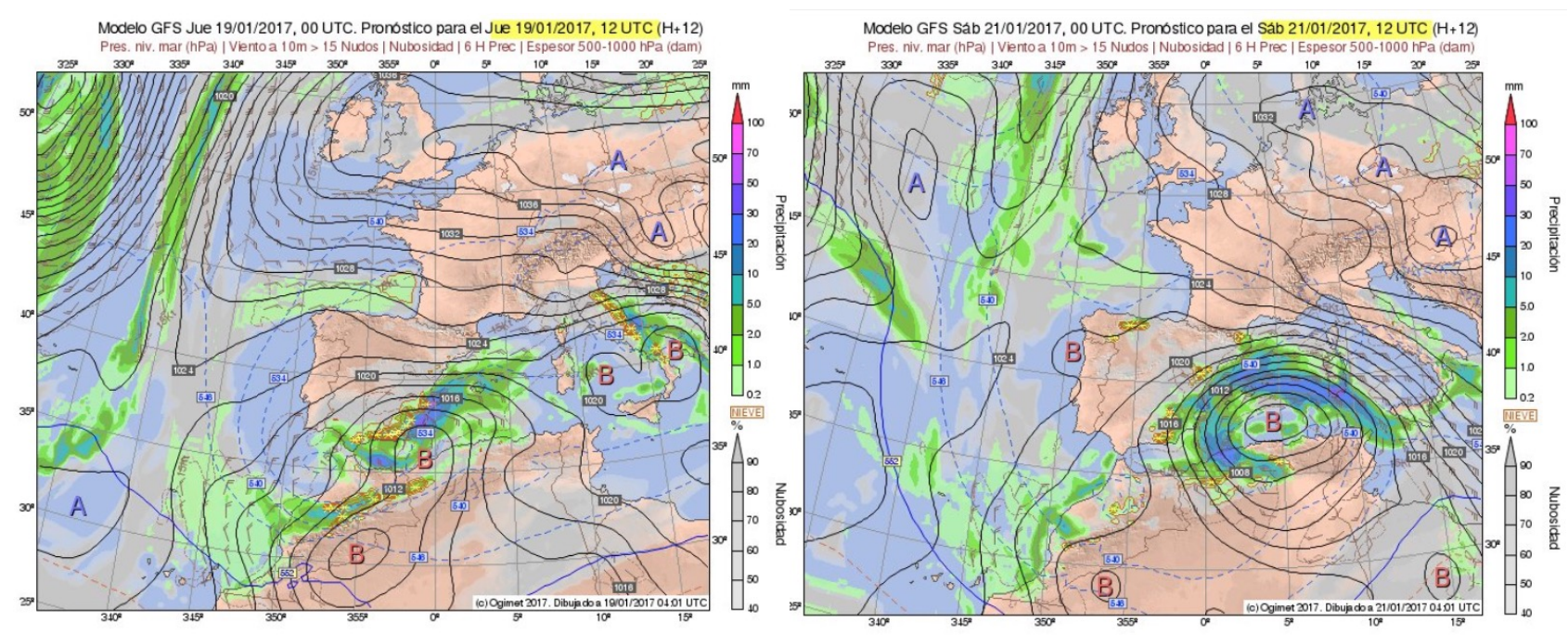

Figura 52.2: Previstos GFS, en superficie, a 12 horas, vía OGIMET (www. ogimet. com), para el día 19 a 12 UTC (izquierda) y el día 21 a 12 UTC (derecha). 

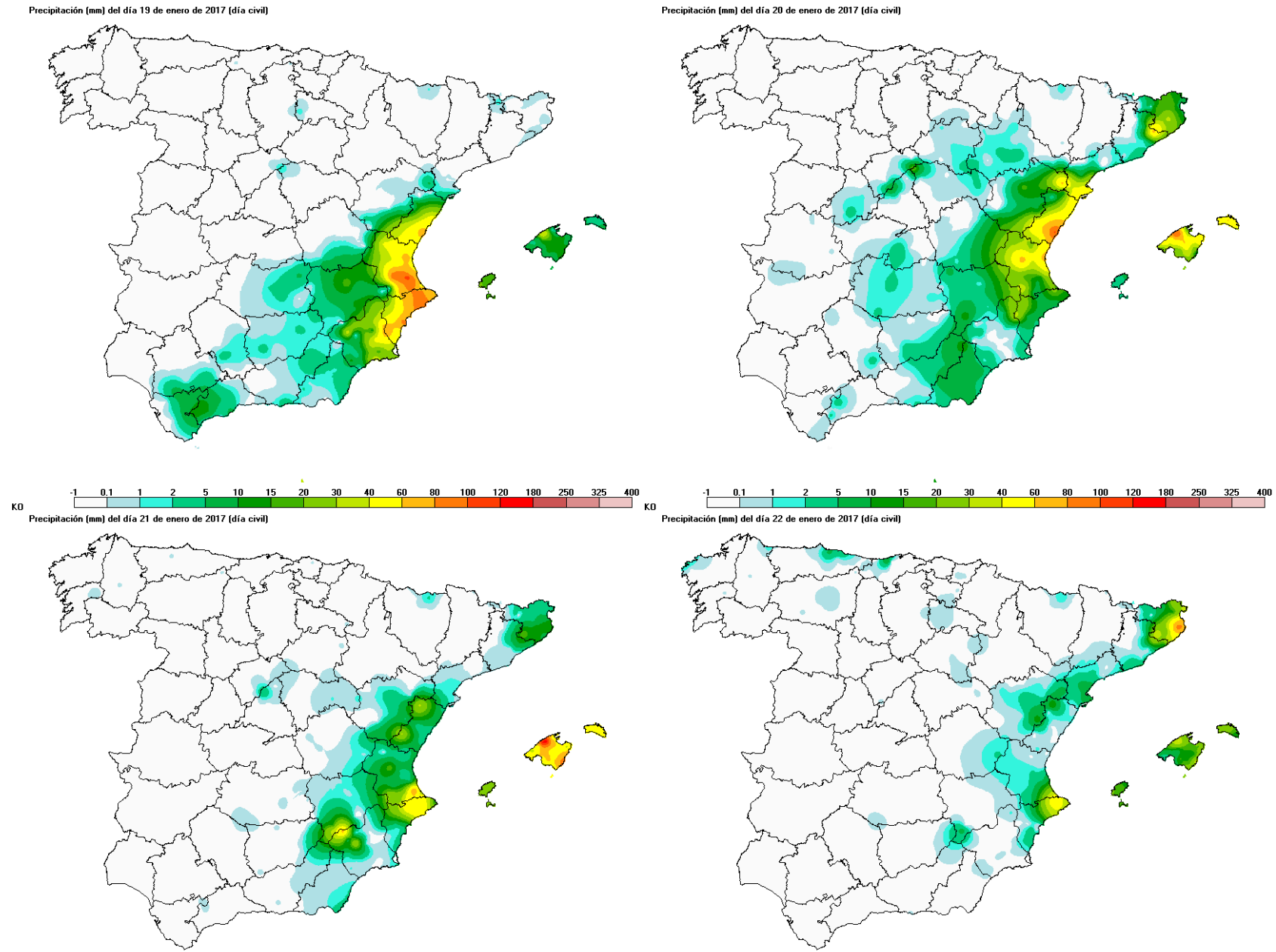

ко
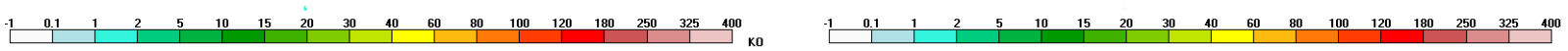

Figura 52.3: Análisis Agencia Estatal de Meteorología (AEMET) de precipitaciones totales en el día civil (de 00 a 24 horas). De izquierda a derecha y de arriba abajo, días 19, 20, 21 y 22 de enero de 2017. Cortesía de César Rodríguez Ballesteros, AEMET.

De acuerdo con lo expuesto en general sobre simultaneidad entre lluvias fuertes y depresiones en el Mediterráneo (cap. 32.2 en la página 535), las dos fases más claramente ciclónicas se corresponden con dos fases distintas de la lluvia intensa, que afectan al día 19 y a los días 21 y 22. El día 20 no es que no haya cierta circulación ciclónica, pero es menos clara y las precipitaciones parecen tener menos organización (ver Figura 52.3).

En la Comunidad Valenciana, en concreto, en este episodio no hay proporcionalidad entre la intensidad y organización de la depresión y la copiosidad de las lluvias. Se cumple aquello de que una depresión incluso débil puede ser suficiente para organizar un efectivo flujo cálido y húmedo, alimentador de la lluvia fuerte e inestabilizador de la atmósfera. En efecto, a pesar de la moderación de la depresión del día 19, las llu- vias ese día son incluso superiores a las de los días 21 y 22, salvo en algún punto muy concreto. El día 20 , en cuanto a lluvias, supone una pausa relativa, si exceptuamos las primeras horas del mismo día 20.

El viento fuerte (y el oleaje alto), en cambio, se corresponden bien con la profundidad de la depresión y son máximos desde la segunda parte del día 21 , cuando la depresión es realmente una profunda borrasca.

Las precipitaciones recogidas en algunas zonas de la Comunidad Valenciana son muy importantes, en particular en las comarcas alicantinas de La Marina Alta y La Marina Baixa y en la valenciana de La Safor, típicas zonas mediterráneas de precipitación intensa ocasional y en las que se localizan los máximos peninsulares en 24 horas [2]. 
Según datos de la organización privada AVAMET (http://www . avamet.org/mx-meteoxarxa. $\mathrm{php}$ ), en algunas estaciones meteorológicas de su red se superaron ampliamente los $600 \mathrm{~mm}$ durante el episodio. En Tàrbena (La Marina Baixa), en particular, se acumularon $668 \mathrm{~mm}$, más de $200 \mathrm{~mm}$ el día 19 y también más de $200 \mathrm{~mm}$ el día 21 . También fueron importantes las precipitaciones en Illes Baleares, pudiéndose destacar el Santuario de Lluc (Serra de Tramuntana, Mallorca), donde el total del episodio (del 19 al 24) arrojó $296 \mathrm{~mm}$, de los que 127 cayeron en un día, el 21.

Llama la atención que muchas de las primeras precipitaciones del episodio y las que se produjeron en los días inmediatamente anteriores fueron en forma de nieve, incluso a cotas bajas, en particular en las comarcas de Alacant i València, donde AEMET tuvo vigente, durante un tiempo, un aviso rojo por nevadas.

La Figura 52.3 en la página anterior, obtenida de análisis AEMET, basados en estaciones de AEMET, permite ver la distribución de las precipitaciones de un modo más global que local. Los mapas corresponden al día civil, de 00 a 24 horas, concretamente a los días 19, 20, 21 y 22. Hay diferencias si se consideran los días pluviométricos (de 08 a 08 horas; no mostrados); en particular, el día 20 hay menos lluvia en la Comunidad Valenciana en el día pluviométrico que en el día civil, lo que confirma la disminución relativa de lluvias a lo largo de día, en esa zona, si exceptuamos la madrugada.

Pese a la moderada magnitud de la depresión implicada, el viento, de componente este, ya fue fuerte el día 19 , pero lo fue más los días 21 y 22, cuando la depresión adquirió toda su intensidad. Tomando como referencia los datos horarios de velocidad media en 10 minutos en la Boya de Dragonera (de Puertos del Estado), situada al suroeste de la isla de Mallorca, el día 19 se llegó a 13,4 m/s y el día 21, 16,6 m/s (alcanzándose la fuerza 8 de la escala Beaufort). El oleaje fue notablemente alto, registrándose alturas significativas superiores a los $6 \mathrm{~m}$, no sólo en Dragonera, sino en diversas boyas de la zona comprendida entre Illes Balears y la península ibérica (Maó, Dragonera, Valencia y cabo de Palos, de Puertos del Estado, y Canal de Eivissa, del Sistema de Observación Costero de las Illes Balears, Sistema de Observación Costero de las Illes Balears (SOCIB), lo que constituye récord en varias de esas boyas. En la boya del Canal de Eivissa (SOCIB), se registraron alturas máximas de oleaje de más de $10 \mathrm{~m}$, lo que también es récord, aunque hay que decir que la serie es corta. Da idea de la fuerza del oleaje que en Cala Rajada, por ejemplo, al nordeste de Mallorca, las olas rompieron el dique del puerto (ver Figura 52.4).

En cuanto a la depresión en sí, no queda claro cómo se originó la primera fase, ni por qué se frustró. Desde luego la región en la que se estableció el mínimo de presión estaba situada bajo una amplia depresión fría en altura, que entre los días 16 y 17 había invadido el Mediterráneo, generando una profunda ciclogénesis, en una zona más bien lejana de la que nos ocupa, hacia el Tirreno.

La ciclogénesis principal dentro de este episodio, ocurrida a lo largo del día 20, hasta el 21, parece haber tenido un carácter muy baroclino, es decir, su energía vendría de la yuxtaposición de masas de aire diferentes, fría y cálida, enroscándose en espiral una sobre la otra, lo cual no significa que no haya habido contribuciones de otros factores, como la liberación de calor latente o los efectos orográficos.

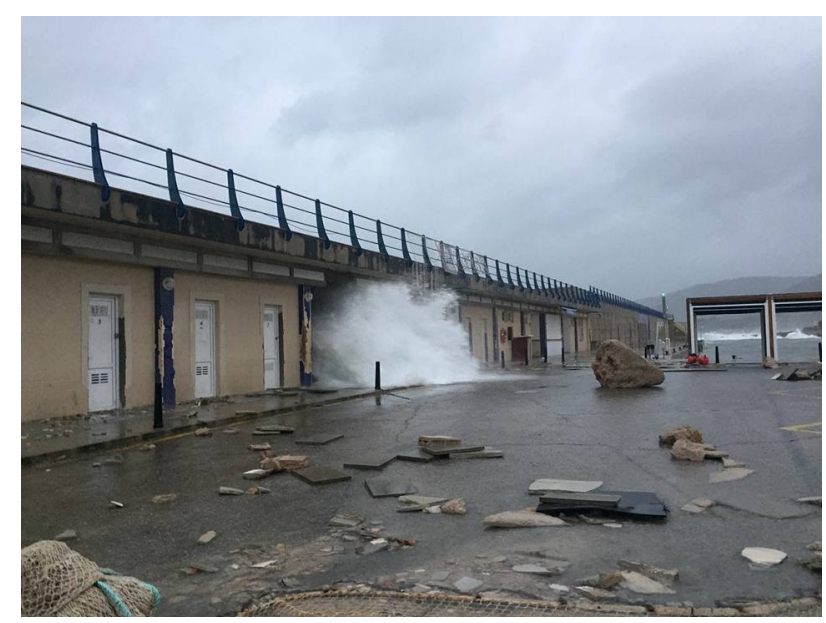

Figura 52.4: Rotura del dique del puerto de Cala Rajada, el 21 de enero de 2017. Fotografía Diario de Mallorca, 21-01-2017, http://www. diariodemallorca. es/part-forana/2017/ 01/21/temporal-rompe-dique-cala-rajada/ 1182946. html. 


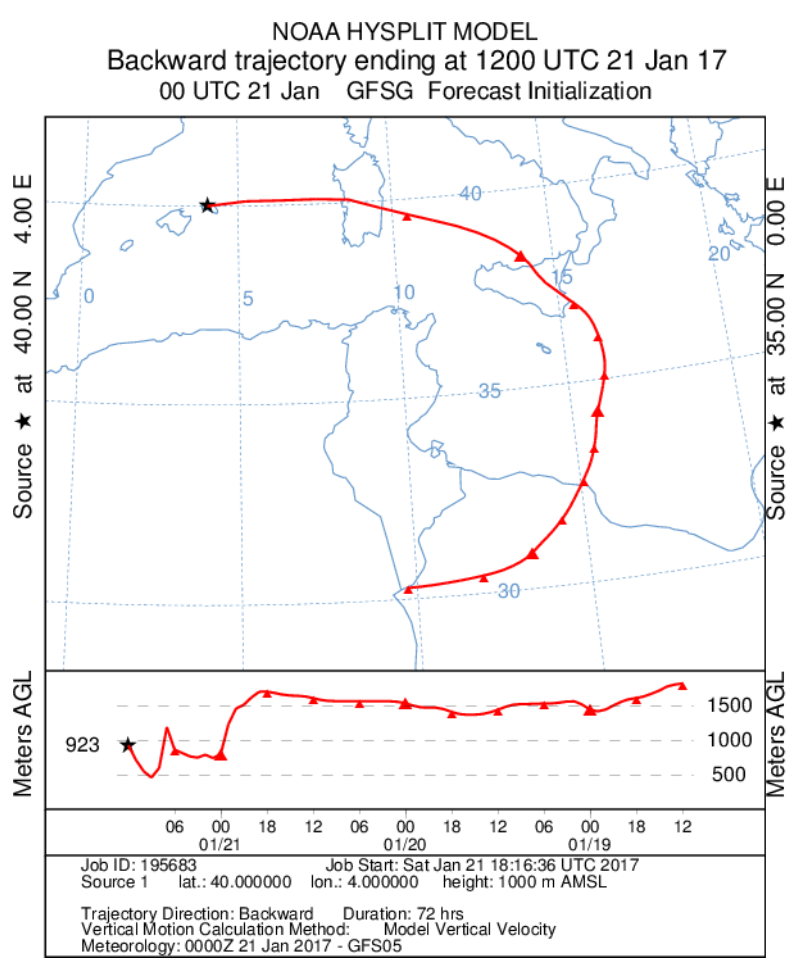

NOAA HYSPLIT MODEL

Backward trajectory ending at 1200 UTC 21 Jan 17 00 UTC 21 Jan GFSG Forecast Initialization

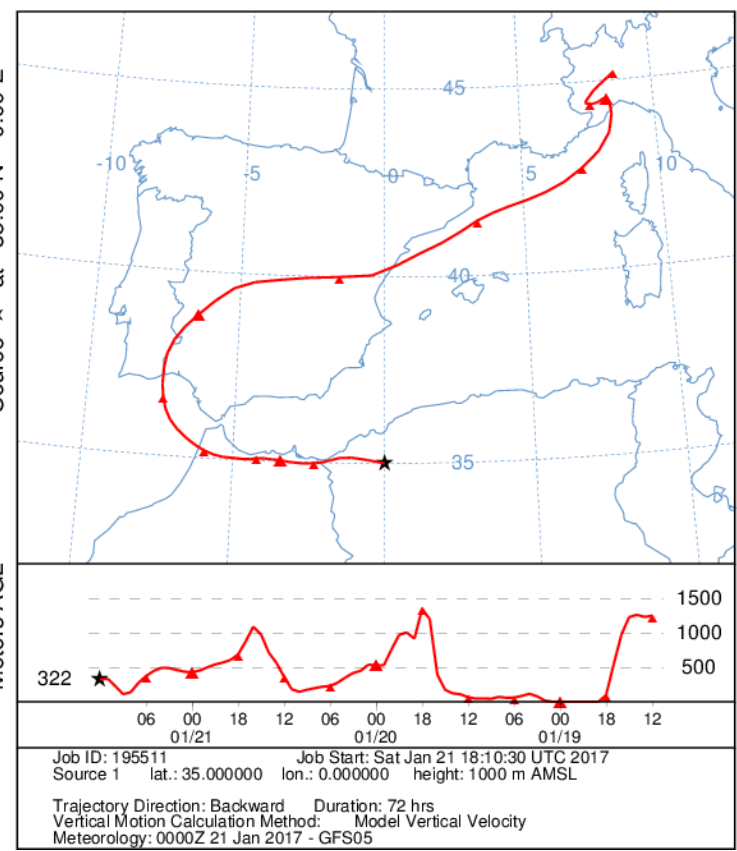

Figura 52.5: Origen de las masas de aire que han entrado en la borrasca mediterránea desde el 19 de enero de 2017 a las 12 UTC, hasta las 12 UTC del día 21. Resultados obtenidos con el método HYSPLIT, de NOAA Air Resources Laboratory [Stein2015].

Mediante el análisis de retrotrayectorias, en la Figura 52.5 puede verse cuál es el origen de las masas de aire que han ido entrando en la borrasca, en formación y ya madura. Aire africano cálido ha entrado hacia el norte de la borrasca, por el sur y el este, mientras aire frío de procedencia europea (resultado de la entrada fría que había habido desde los días 16 y 17) gira, desde el oeste, hacía el sur de nuestra borrasca.
Las imágenes de satélite, en canal de vapor de agua (Canal 5), a las 12 UTC y a las 21 UTC de día 21, visualizan el enroscamiento de las masas de aire; el aire frío seco, en descenso, se corresponde con zonas oscuras, mientras el aire cálido, húmedo, en ascenso, se ve en tonos claros, blancos. La borrasca llega a tener un aspecto incluso amenazador (Figura 52.6).
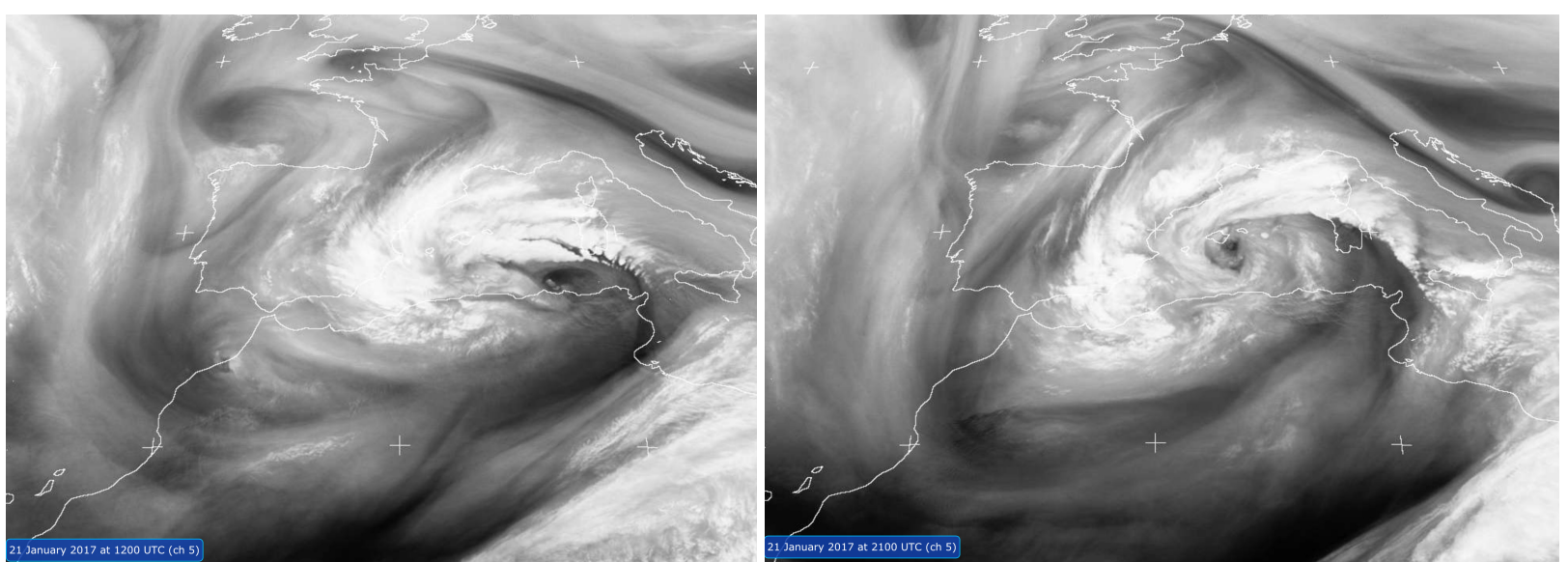

Figura 52.6: Imágenes MSG (sec. 7.3 en la página 86), en canal 5 (vapor de agua), día 21 de enero de 2017, a las 12 UTC (izquierda) y a las 21 UTC (derecha). European Organisation for the Exploitation of Meteorological Satellites (EUMETSAT), vía Universidad de Dundee, UK. 

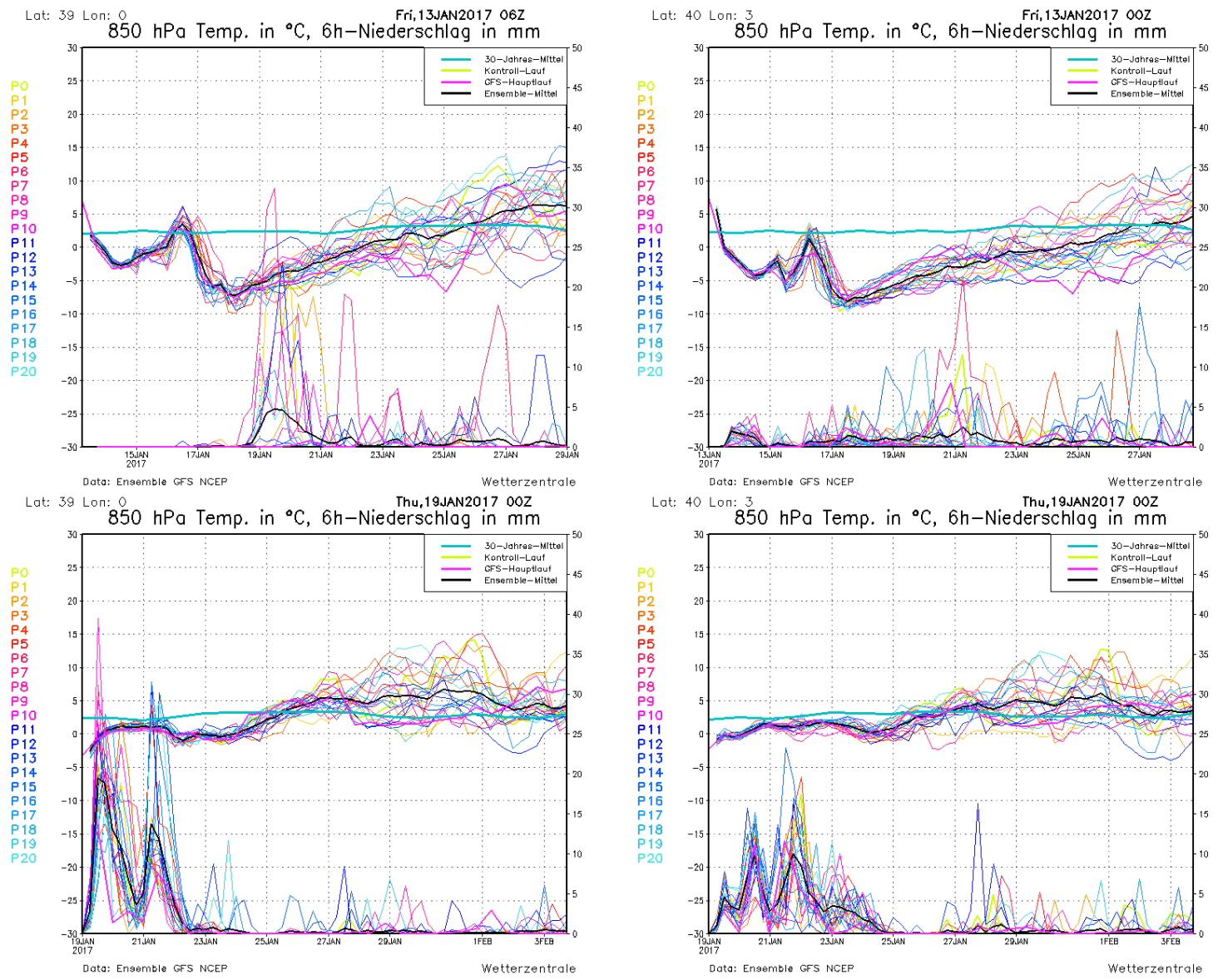

Figura 52.7: Predicciones de precipitación y temperatura en $850 \mathrm{hPa}$, iniciadas el 13 (arriba) y el 19 (abajo) de enero de 2017, en $39^{\circ} \mathrm{N} 0^{\circ} \mathrm{E}$ (izquierda) y en $40^{\circ} \mathrm{N} 3{ }^{\circ} \mathrm{E}$ (derecha), procedente del sistema GEFS-NOAA [4, 5, 6] de predicción por conjuntos. Línea azul turquesa: media climatológica; negra: media del conjunto, rosa fucsia: pasada de control, verde claro: pasada determinista principal. (Adaptado de la presentación online de http: //www. wetterzentrale. de/).

\subsection{Predicciones probabilistas}

\subsubsection{Penachos GEFS}

La Figura 52.7 muestran resultados de la predicción por conjuntos basada en el SPC GEFS [6] de NOAA, en una de las formas en que los presenta http://www. wetterzentrale.de/, en puntos de rejilla (malla) seleccionados. Los puntos seleccionados son $39^{\circ} \mathrm{N} 0^{\circ} \mathrm{E}$, cerca de la costa sur de la provincia de Valencia, y $40^{\circ} \mathrm{N} 3{ }^{\circ} \mathrm{E}$, en el nordeste de Illes Balears. Son predicciones de temperatura a $850 \mathrm{hPa}$ y precipitación, cada seis horas, con inicio día 13 (Figura 52.7, arriba) y día 19 (Figura 52.7, abajo).

Analizando la Figura 52.7, se puede decir que, a dos días vista, es muy clara la caída de temperatura en
$850 \mathrm{hPa}$, con muy poca incertidumbre, tanto en Valencia, como en Illes Balears. En Valencia es, además, relativamente probable lluvia fuerte en la zona el día 19, lo que sería una indicación válida, a cuatro días vista. La media del conjunto de predicciones no da mucha agua para día 19, pero algunos elementos del conjunto sí, particularmente la pasada de control y la determinista del GFS. Desde entonces, la precipitación en Valencia es ruidosa y relativamente poco importante, incluido el día 21: no hay apenas información útil. En Illes Balears, alguna idea de lluvia importante día 21 sí la hay, aunque todo el período de predicción sufre bastante dispersión (ver sección 27.5.1 en la página 412). Las predicciones iniciadas el día 19 dan indicaciones claras del comportamiento de las precipitaciones, tanto en Valencia, como en Illes Balears, para los días 19, 20 y 21. Al acercarse el período de tiempo revuelto, la incertidumbre ha ido disminuyendo. 


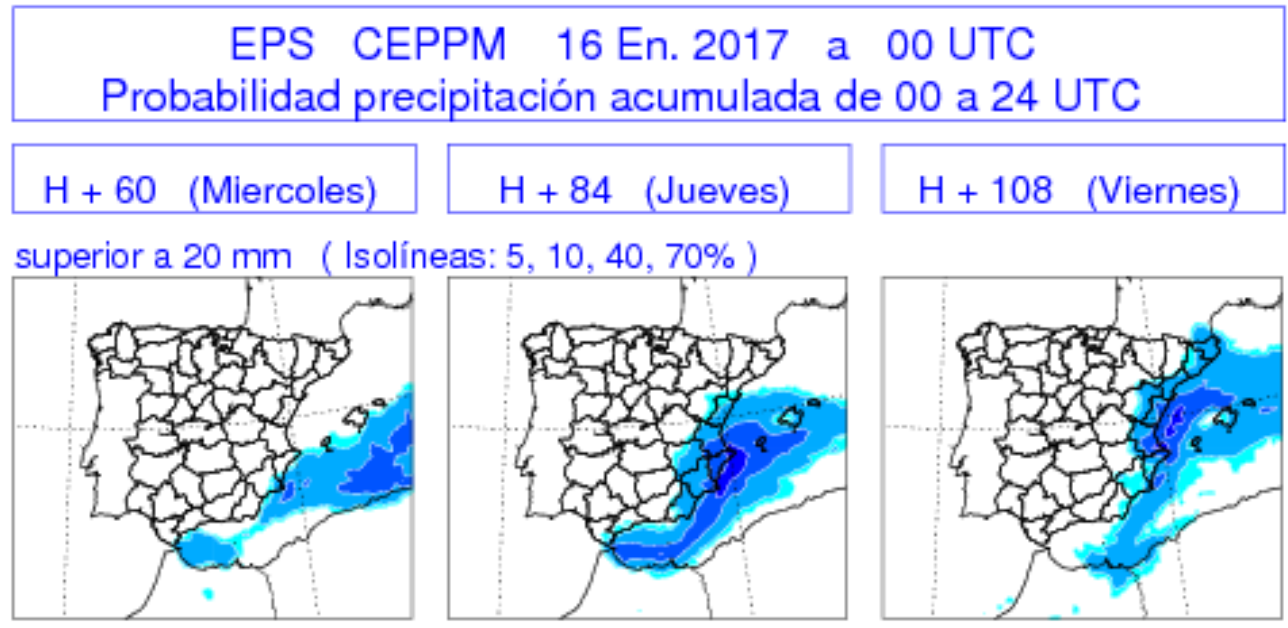

Figura 52.8: Probabilidad de precipitación acumulada en 24 horas superior a 20 mm, según ECENS, para los días 18, 19 y 20 de enero de 2017 (de izquierda a derecha). AEMET 2017.

\subsubsection{Mapas de probabilidad ECENS}

Otra manera de analizar el comportamiento de las predicciones de precipitación es a través de los mapas de probabilidad de sobrepasar un umbral determinado (sección 27.6.1 en la página 416). Para ello se han usado las predicciones por conjuntos del Centro Europeo (ECENS; no tendrían por qué coincidir con las del GFS en pequeñas escalas) y se ha considerado el umbral de $20 \mathrm{~mm}$ en 24 horas, en el día civil correspondiente.

En la Figura 52.8 aparecen las probabilidades de lluvia superior a $20 \mathrm{~mm}$ para los días 18,19 y 20, según predicción del 16 de enero de 2017 a las 00 UTC, del sistema de predicción por conjuntos del Centro Europeo (ECENS). A 84 horas vista hay una clara indicación, con muy poca incertidumbre, de precipitación fuerte el día 19 (sábado), entre el sur de Valencia y Alicante. También hay una disminución clara de precipitación en esa zona concreta para el día 20 (domingo).

En la Figura 52.9 se analizan las predicciones probabilistas de precipitación para día 21 (sábado), a 132 horas vista (incluyendo los días 22 y 23 ) y a 84 horas vista (incluyendo los días 20 y 22). Al plazo más largo la indefinición de la predicción de precipitación para día 21 es mucha: hay una amplia zona con una misma probabilidad de superar los $20 \mathrm{~mm}$, que, además, no es alta, entre el 10 y el $40 \%$. Al aproximarse la fecha, como es de esperar, la incertidumbre disminuye claramente y la predicción probabilista se hace más útil y, además, bastante congruente con lo sucedido luego.
Hay que reconocer que decir que hay una alta probabilidad de más de $20 \mathrm{~mm}$ tampoco es afirmar que vayan a caer 100 o $200 \mathrm{~mm}$, pero sí debe destacarse que la superación de umbrales altos es más significativa que la de umbrales bajos, dadas sus diferentes distribuciones climatológicas. En cualquier caso, es interesante comparar las Figuras 52.8 y 52.9 con la Figura 52.3 en la página 797, con las reservas necesarias en cuanto a la interpretación de la información mostrada en cada figura.

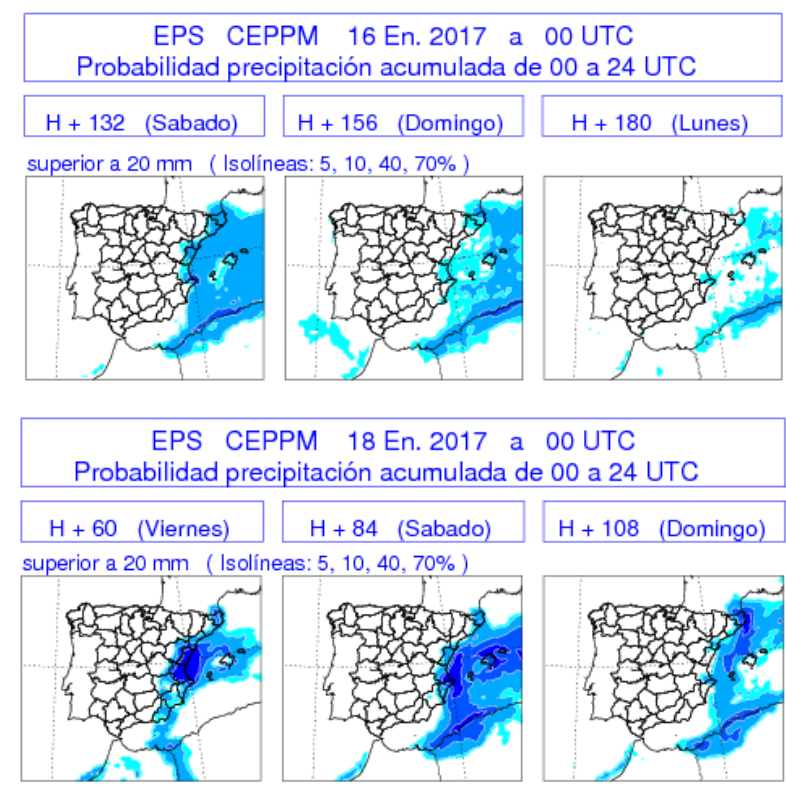

Figura 52.9: Probabilidad de superar $20 \mathrm{~mm}$, según predicciones ECENS iniciadas el 16 (arriba) y el 18 (abajo) de enero de 2017 a 00 UTC, para el sábado 21 y los días inmediatamente próximos. (ECMWF; elaboración, AEMET). 
MAPAS DE PROBABILIDAD // Pasada: 16 En. 2017 a 00 UTC Altura de olas mar de viento

$\mathrm{H}+108$ (Viernes) $\mathrm{H}+132$ (Sabado) $\mathrm{H}+156$ (Domingo)

Altura olas mar de viento superior a $4 \mathrm{~m}(2,5,10,40,70)$

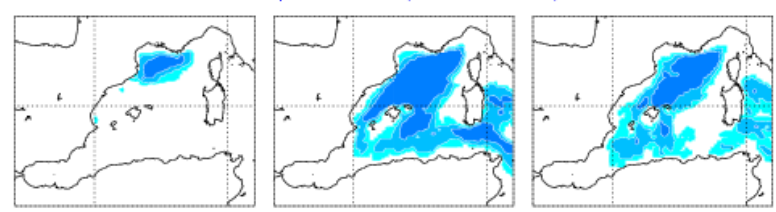

MAPAS DE PROBABILIDAD // Pasada: 18 En. 2017 a 00 UTC Altura de olas mar de viento

$\mathrm{H}+36$ (Jueves) $\mathrm{H}+60$ (Viernes) $\mathrm{H}+84$ (Sabado)

Altura olas mar de viento superior a $4 \mathrm{~m}(2,5,10,40,70)$

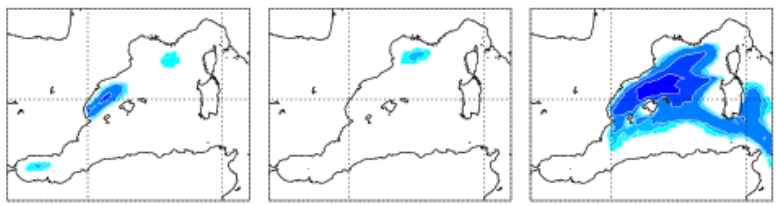

Figura 52.10: Probabilidad de superar los $4 \mathrm{~m}$ de altura significativa de olas, según predicciones ECENS iniciadas el 16 (arriba) y el 18 (abajo) de enero de 2017 a OO UTC, para el sábado 21 y los días inmediatamente próximos. (ECMWF; elaboración, AEMET).

La utilidad de la predicción de vientos y mar se considera aquí analizando las predicciones de oleaje directamente, ya que las predicciones de oleaje son, como es sabido, totalmente dependientes de las prediccio- nes de viento. Ver Figura 52.10. Recuérdese que se observaron alturas superiores a $6 \mathrm{~m}$ en boyas situadas entre las Illes Balears y las costas de Valencia, hasta Murcia (cabo de Palos). La probabilidad de superar los $6 \mathrm{~m}$ es nula en las predicciones del ECENS. En buena parte de la zona citada, la probabilidad de superar, no los 6, sino los $4 \mathrm{~m}$ es aún muy baja, atendiendo a las predicciones a 132 horas vista. Con predicciones a plazo más corto (84 horas) la probabilidad aumenta, pero no en toda la zona, ni de un modo muy rotundo: la predicción se hace más indicativa, más útil, pero no llega a ser plenamente satisfactoria.

Cabría interpretar que la predicción de vientos ha sido insuficiente y ello podría ser debido a que la ciclogénesis principal que define buena parte de ese episodio, no ha sido plenamente prevista por el ECENS, o, al menos, no ha sido bien prevista por un porcentaje importante de los elementos del conjunto.

Considerando las predicciones a 84 horas, la Figura 52.11 compara los mapas medios asociables a dos grupos (ver sección 27.4.1 en la página 406) de elementos de la predicción por conjuntos ECENS (agrupación por semejanza). En el grupo 2 (13 miembros) hay más gradiente de presión, más viento, que en el grupo 3 (11 miembros), pero quizás el gradiente sea insuficiente en ambos grupos de predicciones; quizás también en el primero, no mostrado.
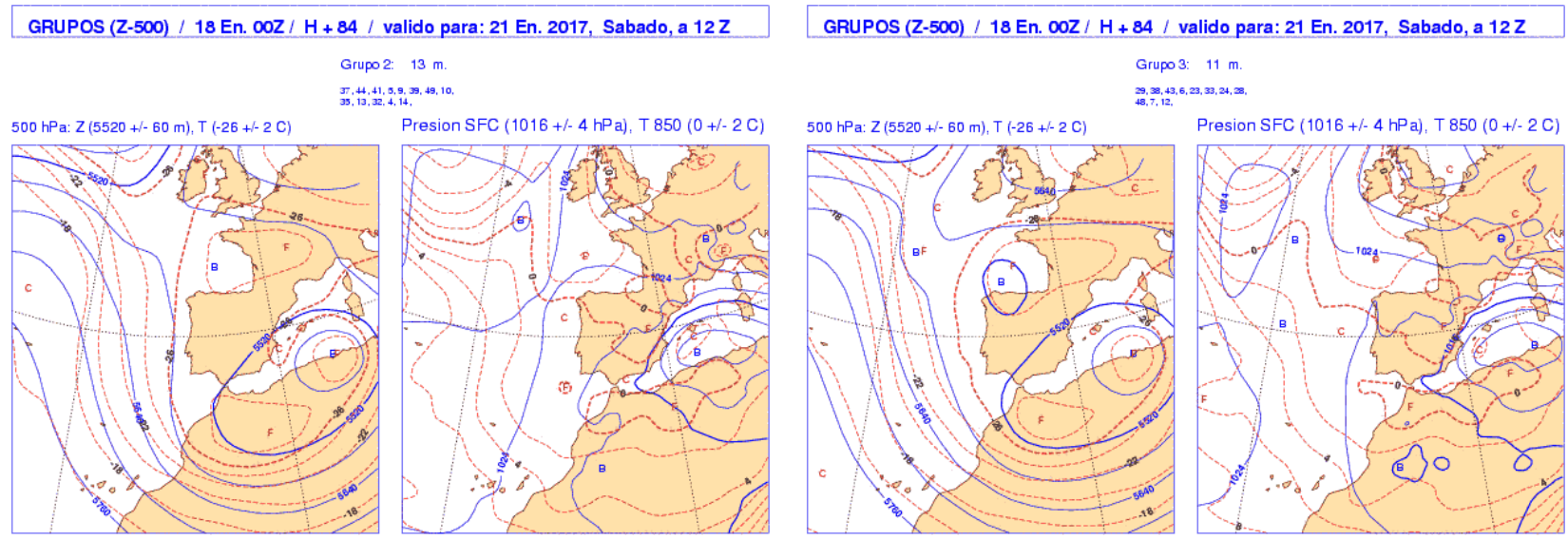

Figura 52.11: Dos grupos de predicciones a 84 h del ECENS, validas para el 21 de enero de 2017 a las 12 UTC, con 13 y 11 elementos respectivamente. En cada grupo (pareja de mapas) puede verse la predicción media de geopotencial a $500 \mathrm{hPa}$ (izquierda) y presión en superficie (derecha) (ECMWF; elaboración, AEMET). 
Más débil es la borrasca que dibujan las predicciones de grupo 2 (12 miembros) de la predicción a 132 horas (Figura 52.12). Las diferencias de la predicciones de presión en superficie parecen asociables a diferencias en la predicción de geopotencial al nivel de $500 \mathrm{hPa}$.

Parece claro que en la ciclogénesis de 20-21 de enero de 2017 los factores orográficos, que serían los que más podrían aumentar la predecibilidad en casos de formación de borrascas, no han sido los más importantes.

Una ciclogénesis mediterránea como la considerada, relevante por su intensidad, más bien baroclina en cuanto al mecanismo de formación, con contribución probable de la liberación de calor latente de condensación y que tiene lugar a una escala espacial bastante menor que las ciclogénesis oceánicas más habituales tiene una limitada predecibilidad. Una herramienta como ECENS puede dar una orientación útil, pero no se puede esperar que aporte resultados plenamente satisfactorios en las escalas necesarias.

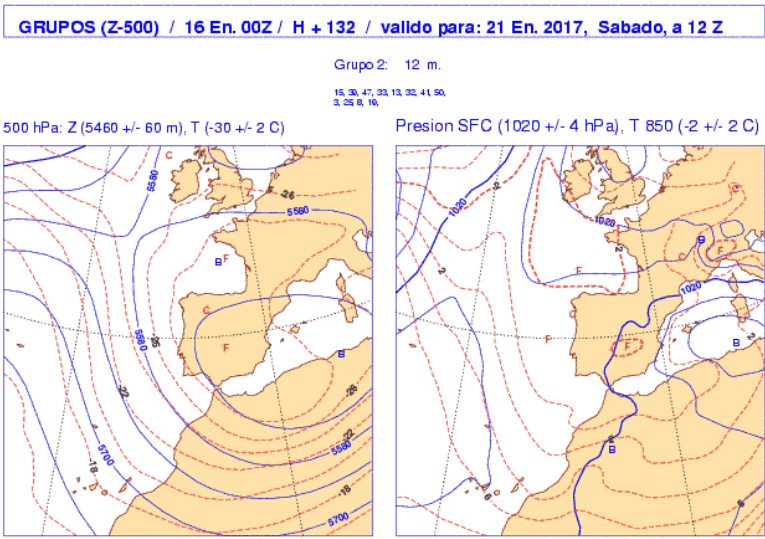

Figura 52.12: Grupo de predicciones a $132 \mathrm{~h}$ del ECENS con 12 miembros, válidas para el 21 de enero de 2017 a las 12 UTC. En cada grupo (pareja de mapas) puede verse la predicción media de geopotencial a $500 \mathrm{hPa}$ (izquierda) y presión en superficie (derecha)(ECMWF; elaboración, AEMET) 


\subsection{Referencias}

[1] Bermejo, N. Cold wave along the Spanish Mediterranean coast - EUMETSAT. Informe técnico. EUMETSAT, 2017. URL: https : //www . eumetsat . int/website/home/ Images / ImageLibrary / DAT_ 3639834 . html (citado en página 795).

[2] Font Tullot, Inocencio. Climatología de España y Portugal. Volumen 76. Universidad de Salamanca, 2000 (citado en página 797).

[3] SELA, Joseph G. "Spectral modeling at the national meteorological center". En: Monthly Weather Review 108.9 (1980), páginas 1279-1292 (citado en página 796).
[4] SELA, Joseph G. "The NMC spectral model". En: NOAA Technical Report, NWS-30 108 (1982) (citado en páginas 796, 800).

[5] Toth, Zoltan y KaLnAY, Eugenia. "Ensemble forecasting at NMC: The generation of perturbations". En: Bulletin of the american meteorological society 74.12 (1993), páginas 2317-2330 (citado en página 800).

[6] Tотн, Zoltan y KaLnAY, Eugenia. "Ensemble forecasting at NCEP and the breeding method". En: Monthly Weather Review 125.12 (1997), páginas 3297-3319 (citado en página 800$)$. 
